Conclusions Serum CIC and IL-6 significantly correlated with clinical SLEDAI, which is higher degree of correlation than anti-dsDNA, C4 and C3 levels. Our study suggested that CIC and IL-6 can be used as alternative biomarkers to determine SLE activity.

\section{IMMUNE COMPLEX GLOMERULONEPHRITIS ASSOCIATED WITH LYSINURIC PROTEIN INTOLERANCE: A CASE REPORT AND REVIEW OF THE LITERATURE}

${ }^{1} \mathrm{MH}$ Tseng*, ${ }^{2} \mathrm{~L}$ Shih-Hua, ${ }^{1} \mathrm{H}$ Jing-Long. 'Chang Gung Memorial Hospital, Paediatrics, TAOYUAN, Taiwan R.O.C; ${ }^{2}$ Tri-Service General Hospital, Medicine, Taipei, Taiwan R.O.C

\subsection{6/lupus-2017-000215.324}

Background and aims Patients with lysinuric protein intolerance (LPI) due to inherited defect of cationic amino acid transport in intestine and renal tubules may have aberrant immune responses leading to multiple organ involvement. The renal involvement with immune complex glomerulonephritis in LPI is albeit rare and has not been well established.

Methods We report a 4-year-old boy manifested nephrotic syndrome with renal histological findings showing immune complex glomerulonephritis highly suggested of lupus nephritis, but the initial serology survey excluded the diagnosis of SLE initially. The diagnosis of lysinuric protein intolerance was established and SLE developed 1 year later. Renal manifestations in patients with LPI and the coexistence of LPI with SLE are reviewed.

Results The initial renal involvement in LPI included renal tubular dysfunction, nephritic and nephrotic syndrome. During follow-up, some patients developed renal function impairment and may progress to end stage renal disease. Glomerulus was the major involved lesion with the most common histological finding was immune complex glomerulonephritis. Five patients, including our patient, with LPI coexisted with SLE have been reported during follow-up. These patients characterised female predominant, young onset age, predominant renal involvement, and poor prognosis. Our patient supported the suggested mechanism of macrophage activation. Treatment with steroid and cyclosporine accordingly led to remission of nephritis.

Conclusions LPI was not only a disorder of amino acid wasting but also a complex multisystemic disease with aberrant immune responses. LPI-associated glomerulonephritis shares similar characteristics on renal histology with lupus nephritis. Both macrophage activation and excess arginine accumulation might play roles on the pathogenesis.

\section{IS LUPUS AN INFLAMMATION RESOLUTION DEFICIENCY DISORDER?}

${ }^{1} D$ Undurti*. ${ }^{1}$ UND Life Sciences LLC, R \& D, Federal way, USA

10.1136/lupus-2017-000215.325

Background and aims Lupus and lupus nephritis progression and flares are difficult to predict. Recently, osteoprotegerin, endothelin-1, $\mathrm{CXCR}^{+}{ }^{+} \mathrm{CD}^{+}{ }^{+} \mathrm{T}$ cells and MCP-1 mRNA expression in urine sediments have been described as possible biomarkers of lupus and lupus nephritis. But their relationship with histological activity has not been sufficiently explored. It is desired that biomarkers of a disease should more rapidly reflect disease progression which would allow shorter clinical proof of concept trials and should be able to predict flares, measure current disease activity and severity, predict progression of disease and prognosis.

Methods It is likely that continued inflammatory events seen in lupus could be due to failure of the resolution of inflammation. Thus, the balance between inflammation and resolution is tilted more in favour of pro-inflammatory events and/ or failure of production of pro-resolution molecules at the most appropriate time leading to non-resolution of inflammation. One such endogenous pro-resolution and anti-inflammatory molecule is lipoxin $\mathrm{A}_{4}$, whose deficiency could lead to continuation of inflammation in lupus and lupus nephritis.

Results It was noted that low plasma and urinary lipoxin $\mathrm{A}_{4}$ indicated disease activity and progression of disease, while a fall in its levels were noted prior to impending flares and increase in disease activity; and an increase in the levels of lipoxin $\mathrm{A}_{4}$ suggested resolution of inflammation and amelioration of disease process.

Conclusions It is suggested that measurement of plasma and urinary lipoxin $\mathrm{A}_{4}$ will be a good biomarker to predict flares, measure current disease activity and severity, predict progression of disease and prognosis.

\section{ROLE OF BIOACTIVE LIPIDS IN AUTOIMMUNE DISEASES INCLUDING LUPUS}

${ }^{1}$ D Undurti*. ${ }^{1}$ UND Life Sciences, $R \& D$, Federal Way, USA

10.1136/lupus-2017-000215.326

Background and aims To evaluate whether a regimen of corticosteroids and cyclophosphamide, and methods designed to enhance endothelial NO synthesis and augment antioxidant defenses can lead to long-lasting remission of lupus and other autoimmune diseases.

Methods Patients with established lupus, dermatomyositis and rheumatoid arthritis are seelcted for the study. Their plasma phospholipid content of unsaturated fatty acids, nitric oxide, anti-oxidants and cytokines are measured before and after treatment.

Results Plasma phospholipid content of arachidonic, eicosapentaenoic and docosahexaenoic acids (AA, EPA and DHA respectively) were found to be low and so also plasma nitric oxide levels and anti-oxidants with a concomitant increase in plasma IL-6 and TNF concentrations. These patients were given pulses of methylprednisolone and cyclophosphamide based on their disease status and response to therapy followed by oral supplementation of GLA/EPA/DHA daily.

Conclusions All patients who entered the study went in to full remission that was found to be associated with restoration of plasma phospholipid content of AA/EPA/DHA, antioxidants and nitric oxide and cytokines to normal. All these patients were in full remission even after stopping immunosuppressive drugs but are continuing GLA/EPA/DHA orally. 